\title{
Clinical significance of tumor markers in detection of recurrent hepatocellular carcinoma after radiofrequency ablation
}

\author{
TETSUYA BEPPU $^{1}$, KAZUSHI SUGIMOTO ${ }^{1}$, KATSUYA SHIRAKI ${ }^{1}$, MASAHIKO TAMEDA $^{1}$, \\ SATOKO KUSAGAWA $^{1}$, KEIICHIRO NOJIRI ${ }^{1}$, JUNICHIRO TANAKA ${ }^{1}$, NORIHIKO YAMAMOTO ${ }^{1}$, \\ YOSHIYUKI TAKEI ${ }^{1}$, HARUYUKI TAKAKI ${ }^{2}$, JUNJI URAKI ${ }^{2}$, ATSUHIRO NAKATSUKA ${ }^{2}$, \\ KOICHIRO YAMAKADO ${ }^{2}$ and KAN TAKEDA ${ }^{2}$
}

Departments of ${ }^{1}$ Gastroenterology and ${ }^{2}$ Radiology, Mie University School of Medicine, Tsu, Mie 514-8507, Japan

Received March 19, 2010; Accepted May 10, 2010

DOI: 10.3892/ijmm_00000482

\begin{abstract}
The aim of this study was to elucidate the importance of three tumor markers, $\alpha$-fetoprotein (AFP), Lens culinaris agglutinin A-reactive fraction of AFP (AFP-L3), and des- $\gamma$-carboxy prothrombin (DCP), for detecting and predicting the recurrence of hepatocellular carcinomas (HCCs). A total of 108 patients with initial non-advanced HCC who underwent curative radiofrequency ablation (RFA) in our hospital were enrolled in this study. The effectiveness of the three tumor markers for detecting recurrence and recurrence-free survival was analyzed. Positivity of these three makers was not markedly increased at the first or second recurrence. In addition, there was no significant correlation between the initial and recurrent levels of each tumor marker. The tumor marker that was positive at the time of initial HCC was not necessarily positive at recurrence. The tumor marker levels at recurrence were not correlated with pre-ablation levels. No significant correlation was found in the tumor marker values between pre-ablation and the time of recurrence. On multivariate analysis, high AFP-L3 levels $(\geq 10 \%)$ were significantly predictive of recurrence-free survival. All three tumor markers should be routinely measured to detect recurrence during follow-up after RFA. Especially high AFP-L3 levels should be followed closely.
\end{abstract}

\section{Introduction}

Hepatocellular carcinoma (HCC) is one of the most common malignancies worldwide, and its incidence is still increasing in various regions (1-3). Current options for the treatment of

Correspondence to: Dr Katsuya Shiraki, Department of Gastroenterology, Mie University School of Medicine, 2-174 Edobashi, Tsu, Mie 514-8507, Japan

E-mail: katsuyas@clin.medic.mie-u.ac.jp

Key words: hepatocellular carcinoma, radiofrequency ablation, $\alpha$-fetoprotein, Lens culinaris agglutinin A-reactive fraction of AFP, des- $\gamma$-carboxy prothrombin
HCC consist of surgical resection, orthotropic liver transplantation, transcatheter arterial embolization (TACE) and percutaneous ablation therapy. Surgical resection has been considered to be the best treatment for early-stage HCCs, but only $9-29 \%$ of patients with HCC are candidates for surgery, owing to either underlying chronic liver disease resulting in poor hepatic reserve or a multifocal distribution of tumor nodules (4). Radiofrequency ablation (RFA) was first described by Rossi et a (5) and it is a relatively new technique for local HCC therapy, with many studies having demonstrated its clinical utility and safety (6-8). A high local cure rate can be achieved without adversely affecting background liver function, and it has been playing an important role in the treatment of HCC (9).

We have shown that RFA combined with chemoembolization provides overall and recurrence-free survival rates comparable to those achieved with hepatectomy in patients with early-stage HCC (4). The local tumor progression rate following RFA combined with chemoembolization (combination therapy) was lower than the rates in previous studies that used RFA alone (10-12). Despite good control of treated lesions, new tumors frequently occur, resulting in high distant recurrence rates $(4,6)$.

Therefore, it is very important to detect recurrence of HCCs early. For this purpose, serum tumor markers have been used for a long time because of their convenience, inexpensiveness, and their satisfactory accuracy, and they could be valuable supplementary investigations to ultrasonography and computer tomography (CT) for the diagnosis of HCC. Using an appropriate single tumor marker or combination of tumor markers may improve the effectiveness of screening HCC patients (13).

Several tumor markers for $\mathrm{HCC}$ have been reported (14-20). Three tumor markers specific to HCC are currently used in Japan, AFP, AFP-L3 and DCP, which is also referred to as protein induced by vitamin K absence II (PIVKA II) (21).

Previous reports have detailed the usefulness of each of these tumor markers in the early detection and diagnosis of $\mathrm{HCC}$, the evaluation of tumor progression, and the determination of patient prognosis (21-26), although these tumor markers do not demonstrate high positivity in patients with small (e.g., less than $2 \mathrm{~cm}$ in maximum diameter) HCC $(27,28)$. Of these, AFP shows relatively low specificity $(29)$, 
Table I. Baseline characteristics of the patients.

\begin{tabular}{lc}
\hline Variable & All patients $(\mathrm{n}=108)(\%)$ \\
\hline Age (years) & $67.3 \pm 8.4$ \\
Gender (male/female) & $77(71) / 31(29)$ \\
HBsAg positive & $10(9)$ \\
HCVAb positive & $89(82)$ \\
AST (IU/ml) & $52.5(19-157)$ \\
ALT $(\mathrm{IU} / \mathrm{ml})^{\mathrm{a}}$ & $45.5(13-162)$ \\
Child-Pugh class $(\mathrm{A} / \mathrm{B})$ & $83(77) / 25(23)$ \\
Albumin $(\mathrm{g} / \mathrm{dl})^{\mathrm{a}}$ & $3.5(2.2-4.7)$ \\
Total bilirubin $(\mathrm{mg} / \mathrm{dl})^{\mathrm{a}}$ & $0.8(0.3-1.9)$ \\
Prothrombin time $(\%)^{\mathrm{a}}$ & $84.7(56.4-117)$ \\
Platelets (x10 $/ \mathrm{mm}^{3) \mathrm{a}}$ & $9.9(3.2-34.1)$ \\
Maximum tumor size $(\mathrm{cm})^{\mathrm{a}}$ & $2.4(1-5)$ \\
$<3$ & $85(79)$ \\
$\geq 3$ & $23(21)$ \\
No. of tumors & $1(1-4)$ \\
Uninodular & $72(67)$ \\
Multinodular & $36(33)$ \\
\hline
\end{tabular}

aData are expressed as median (range). HBsAg, hepatitis B surface antigen; $\mathrm{HCV}$, hepatitis $\mathrm{C}$ virus; AST, aspartate aminotransferase; ALT, alanine aminotransferase.

because AFP increases in association with hepatocyte regeneration and is also associated with serum alanine aminotransferase activity (9). These studies recommend the simultaneous measurement of these tumor markers in the follow-up of patients with chronic liver diseases. However, only a few studies have examined the significance of tumor markers in the prediction, detection and diagnosis of HCC recurrence after local curative treatment using RFA. Therefore, we investigated the use of these three tumor markers in the detection of recurrent HCCs after curative ablation.

\section{Patients and methods}

Patients. Subjects included 108 patients with initial HCC who underwent RFA after TACE for treatment of HCC between April 2000 and April 2008 at Mie University Hospital. Inclusion criteria were: i) no previous treatment for HCC; ii) a single HCC of $\leq 5 \mathrm{~cm}$ in diameter or up to three HCCs, each of which was $\leq 3 \mathrm{~cm}$ in diameter; iii) liver cirrhosis classified as Child-Pugh class A and B; iv) no vascular invasion; and v) no extrahepatic metastases. Patient background data are summarized in Table I. Of the 108 patients, $77(71 \%)$ were men and $31(29 \%)$ were women, with a mean age of 67.3 years. In all, 89 patients (82\%) were positive for anti-HCV antibody and $10(9 \%)$ patients were positive for HBs antigen. With regard to Child-Pugh classification, $83(77 \%)$ patients were class A and 25 (23\%) were class B. The median (range) levels of asparate aminotransferase (AST), alanine aminotransferase (ALT), albumin (Alb), total bilirubin (T-Bil), prothrombin time (PT) and platelet counts (Plt) were 52.5 IU/1 (19-157), $45.5 \mathrm{IU} / 1$ (13-162), $3.5 \mathrm{~g} /$ dl (2.2-4.7), $0.8 \mathrm{mg} / \mathrm{dl}(0.3-1.9), 84.7 \%$ (56.4-117) and $9.9 \times 10^{4} / \mathrm{mm}^{3}(3.2-34.1)$, respectively.

Diagnosis of HCC was primarily established based on radiological findings and elevated tumor marker values. If a hepatic nodular lesion was found on screening ultrasonography, the patient underwent dynamic CT and/or dynamic magnetic resonance imaging (MRI) for further evaluation. Furthermore, in cases with a liver nodule that showed hyperattenuation in the arterial phase of the dynamic study and washout in the portal or delayed phase or typical hypervascular staining on digital subtraction angiography, the nodule was diagnosed as HCC. When the nodule did not show the abovementioned typical imaging features, a fine-needle aspiration biopsy was carried out for histological examination and diagnosis.

In this study, $72(67 \%)$ patients had a single HCC nodule and $36(33 \%)$ had multiple nodules. The median (range) maximum tumor diameter was $2.4 \mathrm{~cm}(1-5) ; 23$ (21\%) patients had tumors of $\geq 3 \mathrm{~cm}$ and $85(79 \%)$ patients had tumors $<3 \mathrm{~cm}$ (Table I). The median (range) follow-up period was 32.3 (6.1-76) months.

Chemoembolization. All patients underwent chemoembolization prior to RFA. After celiac, superior mesenteric and hepatic arteriography, a 3F micro-catheter (Micro Pheret, William Cook Europe, Bjaevevskov, Denmark) was advanced into the arteries supplying each tumor. The feeding arteries were embolized with gelatin sponge particles (Spongel, Yamanouchi, Tokyo, Japan) after a mixture of 2-8 ml iodized oil (Lipiodol Ultra-Fluid, Mitsui, Tokyo, Japan) and $40 \mathrm{mg}$ of epirubicin hydrochloride (Farmorubicin, Kyowa Hakko, Tokyo, Japan) was injected into the arteries.

Radiofrequency ablation. Because the gelatin sponge remains in the tumor for 2-3 weeks after chemoembolization, RFA was generally performed within 2 weeks after TACE. RFA was performed using a 17-gauge, straight electrode with a 2- or 3-cm exposed tip (Cool-tip single needle, Radionics, Burlington, MA, USA) connected to an RF generator (Cooltip RF generator, Radionics).

If the ablation was thought to be incomplete, the second ablation session was performed the next week to complete therapy. The end-point of RFA was the presence of a welldefined area of non-enhancing tissue, consisting of the treated tumor with a tumor-free margin of at least $5 \mathrm{~mm}$ in the arterial and portal phases on enhanced CT imaging. Patients received additional sessions of ablation until complete ablation was confirmed in each nodule.

Therapeutic effects and diagnosis of recurrence. Contrastenhanced CT was performed to evaluate local therapeutic effects 1 week after RFA to check the efficacy of therapy. Subsequently, contrast-enhanced CT was performed every 
3 months to detect recurrence early. If a hepatic tumor was detected but did not present the typical enhancement pattern on $\mathrm{CT}$, superparamagnetic iron oxide-enhanced magnetic resonance imaging was performed. In this study, 8 cases received anti-viral therapy with interferon after RFA, and $\mathrm{HCV}$ disappeared in three patients. Also, $600 \mathrm{mg}$ of ursodeoxycholic acid were given to most patients. Distant recurrence was defined as the appearance of new tumors at an intrahepatic site distant from the treated tumors. When recurrence was suspected, angiography together with CTAP and CTHA was performed to make a definitive diagnosis.

Measurement of tumor markers. AFP, AFP-L3 and DCP levels were measured in serum samples obtained from each patient at the time of HCC diagnosis. The serum AFP levels were determined by enzyme-linked immunosorbent assay using a commercially available kit (ELISA-AFP, International Reagents, Kobe, Japan). Serum AFP-L3 levels were measured by lectin-affinity electrophoresis coupled with antibodyaffinity blotting (AFP Differentiation kit L, Wako Pure Chemical Industries, Ltd., Osaka, Japan) and expressed as a percentage of the total AFP (AFP-L3 level/total AFP X 100). The serum DCP levels were determined by sensitive enzyme immunoassay (Eitest PIVKA II kit, Eisai Laboratory, Tokyo, Japan) according to the manufacturer's instructions. The cut-off levels for each tumor marker were $100 \mathrm{ng} / \mathrm{dl}$ for AFP, $10 \%$ for AFP-L3 and $40 \mathrm{mAU} / \mathrm{ml}$ for DCP, based on previous reports. AFP-L3 in patients with AFP values $<10 \mathrm{ng} /$ dl was determined as $0 \%$, because AFP-L3 is normally quantifiable only in patients with AFP values above $10 \mathrm{ng} / \mathrm{dl}$.

Statistical analysis. Survival and recurrence-free survival rates were computed using Kaplan-Meier estimates, and the Kaplan-Meier method and log-rank test were used to analyze predictive factors for recurrence-free survival of HCC. To identify independent factors associated with the recurrencefree survival rates, various likely predictors associated with recurrence-free survival on univariate analysis were subjected to multivariate analysis.

The Cox proportional hazards model was used for multivariate analysis. The factors included for analyses were patient aspartate aminotransferase (AST $\geq 52 \mathrm{IU} / 1 /<52 \mathrm{IU} / 1$ ), alanine aminotransferase (ALT $\geq 45 \mathrm{IU} / \mathrm{l} /<45 \mathrm{IU} / \mathrm{l})$, serum albumin $(\mathrm{Alb} \leq 3.5 \mathrm{~g} / \mathrm{dl} />3.5 \mathrm{~g} / \mathrm{dl})$, prothrombin time $(\mathrm{PT}<84 \% / \geq 84 \%)$, platelet counts $\left(\mathrm{Plt}<10 \times 10^{4} / \geq 10 \times 10^{4} / \mathrm{mm}^{3}\right)$, pretreatment AFP-L3 level $(<10 \% / \geq 10 \%)$ and pretreatment DCP level $(<40 \mathrm{mAU} / \mathrm{ml} / \geq 40 \mathrm{mAU} / \mathrm{ml})$. A P-value $<0.05$ was considered statistically significant. Analyses were performed using the StatView statistics package (SAS Institute, Cary, NC, USA).

\section{Results}

Overall recurrence-free survival rate and recurrence rate after initial RFA. The recurrence-free survival rates were 86.2 and $23.2 \%$ at 1 and 5 years, respectively. Of the 108 patients, 2 had local recurrences during the follow-up period. Locally recurrent tumors were treated by TACE followed by RFA. The overall local recurrence rate was $3.5 \%$, and the overall distant recurrence rate was $66.3 \%$ at 5 years (data not shown).
Table II. Tumor marker levels

\begin{tabular}{lc}
\hline Variable & $\mathrm{N}(\%)$ \\
\hline AFP $(\mathrm{ng} / \mathrm{dl})$ & $26(2-3173)$ \\
$<100$ & $87(81)$ \\
$\geq 100$ & $21(19)$ \\
AFP-L3 $(\%)$ & $2.8(0-89)$ \\
$<10$ & $83(77)$ \\
$\geq 10$ & $25(23)$ \\
DCP $(\mathrm{mAU} / \mathrm{ml})$ & $27(1.5-25750)$ \\
$<40$ & $64(59)$ \\
$\geq 40$ & $44(41)$ \\
AFP, ALP-L3, DCP & \\
$<100 \mathrm{ng} / \mathrm{dl},<10 \%$ and $<40 \mathrm{mAU} / \mathrm{ml}$ & $47(44)$ \\
$\geq 100 \mathrm{ng} / \mathrm{dl}$ or $\geq 10 \%$ or $\geq 40 \mathrm{mAU} / \mathrm{ml}$ & $61(56)$ \\
\hline
\end{tabular}

AFP, $\alpha$-fetoprotein; AFP-L3, Lens culinaris is agglutinin A-reactive fraction of $\alpha$-fetoprotein; DCP, des- $\gamma$-carboxy prothrombin.

Pre-treatment positivity for each tumor marker. We defined AFP equal to or $>100 \mathrm{ng} / \mathrm{ml}$, AFP-L3 equal to or $>10 \%$ and $\mathrm{DCP} \geq 40 \mathrm{mAU} / \mathrm{ml}$ as positive. Table II shows the rate of AFP, AFP-L3 and DCP positivity: 21 (19\%) patients were positive for AFP, 25 (23\%) patients were positive for AFP-L3 and $44(41 \%)$ patients were positive for DCP before initial HCC treatment, while 47 (44\%) patients were positive for none of these three markers.

Positive tumor marker rates at HCC recurrence. To verify the importance of three tumor markers for the diagnosis of recurrence, the positivity rates for each tumor marker at the initial diagnosis, first recurrence, and second recurrence were established. AFP was positive in $19 \%, 14 \%$ of patients at the initial diagnosis and first recurrence, then increased to $33 \%$ of patients at the second recurrence. AFP-L3 was positive in $23 \%$ at initial diagnosis, then increased to $33 \%$ at first recurrence, but decreased to $20 \%$ at second recurrence. DCP was positive in $41 \%, 47 \%$ at the initial diagnosis and first recurrence, then decreased to $27 \%$ of patients at the second recurrence (Fig. 1). The number of positive tumor markers did not increase with repeated recurrence (Fig. 2).

Tumor markers pre-ablation and at recurrence. We plotted the values of each tumor marker immediately before ablation and at recurrence. For each tumor marker, no correlation was found between the pre-ablation value and the value at the time of recurrence (AFP, $\mathrm{R}=0.079 \mathrm{P}=0.461$; AFP-L3, $\mathrm{R}=0.064 \mathrm{P}=0.704$; DCP, $\mathrm{R}=0.015 \mathrm{P}=0.893$ ) (Fig. 3).

Interestingly, in the patients who were positive for AFP, AFP-L3 and DCP at the initial HCC, 82, 44 and $42 \%$ were negative for the corresponding marker at the time of recurrence, respectively. Furthermore, in the patients who were negative for AFP, AFP-L3 and DCP at the initial HCC, 18, 26 and $29 \%$, respectively, were positive for the corresponding marker when recurrence was diagnosed (data not shown). 

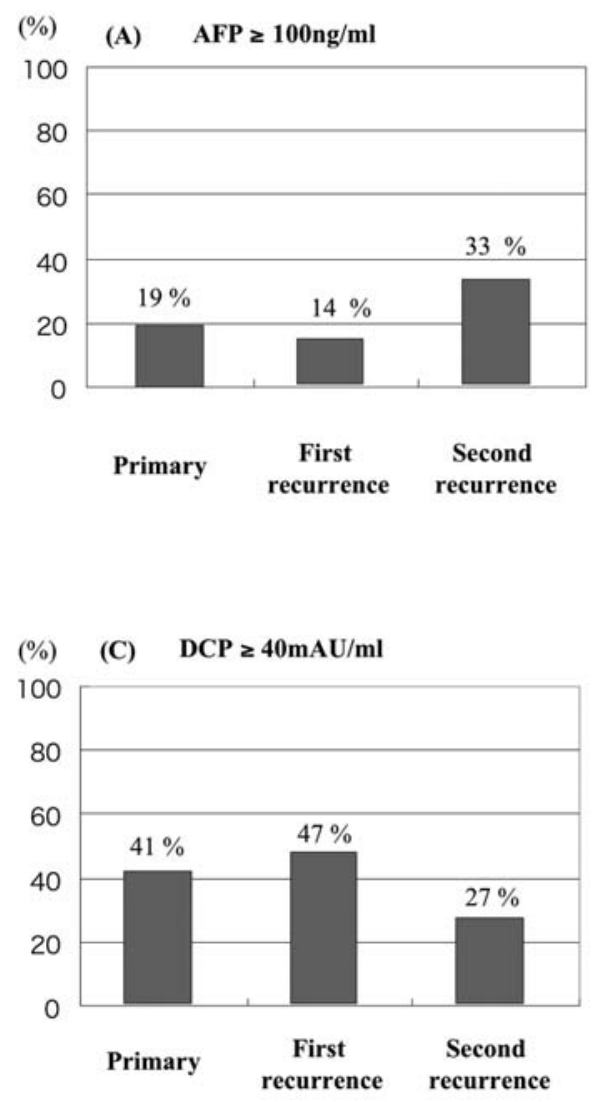

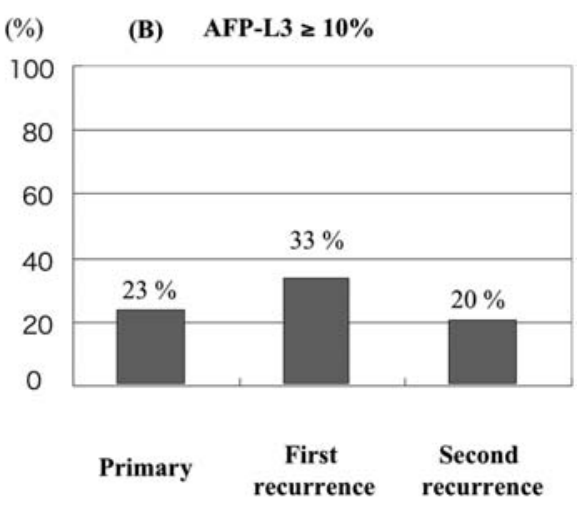

Figure 1. Positivity rates (\%) for each tumor marker [(A) AFP (100 ng/ml), (B) AFP-L3 (10\%), (C) DCP (40 mAU/ml)] at the initial diagnosis, first recurrence and second recurrence. AFP, $\alpha$-fetoprotein; AFP-L3, Lens culinaris agglutinin A-reactive fraction of $\alpha$-fetoprotein; DCP, des- $\gamma$-carboxy prothrombin.

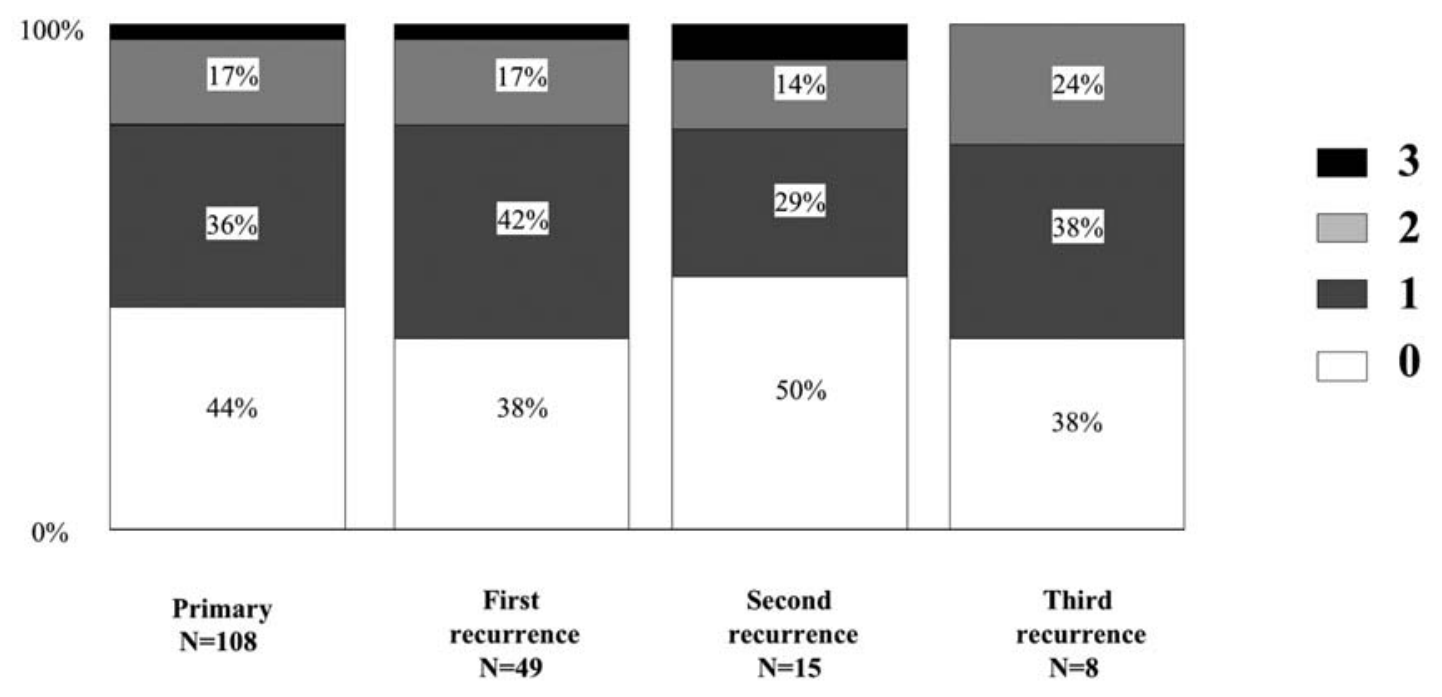

Figure 2. The number (0-3) of positive tumor markers at the initial diagnosis, first, second and third recurrence.

Recurrence-free survival rate after RFA by tumor marker. In the patients with AFP (100 ng/dl, recurrence-free survival rates were 66.6 and $22.2 \%$ at 2 and 4 years, respectively, whereas in the patients with AFP $<100 \mathrm{ng} / \mathrm{dl}$, recurrence-free survival rates were 60.8 and $24.4 \%$ at 2 and 4 years, respectively (NS) (Fig. 4A). In the AFP-L3 (10\% patients, recurrencefree survival rates were 41.9 and $11.2 \%$ at 2 and 4 years, respectively, whereas in the AFP $<10 \%$ patients, recurrencefree survival rates were 67.8 and $26.5 \%$ at 2 and 4 years, respectively $(\mathrm{P}<0.05)$ (Fig. 4B). In the DCP $(40 \mathrm{mAU} / \mathrm{dl}$ patients, recurrence-free survival rates were 44.6 and $20.6 \%$ at 2 and 4 years, respectively, whereas in the DCP $<40 \mathrm{mAU} /$ dl patients, recurrence-free survival rates were 72.0 and $23.3 \%$ at 2 and 4 years, respectively (NS) (Fig. 4C).

Predictive factors for cumulative recurrence-free survival. Table III shows the predictive factors for cumulative recurrencefree survival according to the Kaplan-Meier method in all patients. Significantly lower recurrence-free survival rates were seen in cases with low Alb levels and high AFP-L3 levels 
(A)

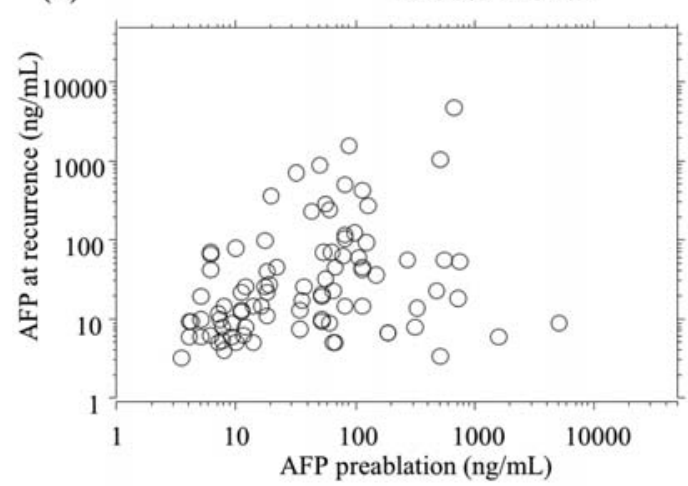

(B)

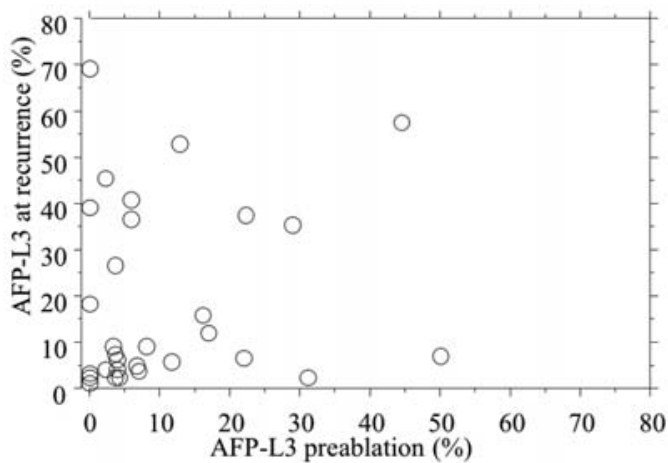

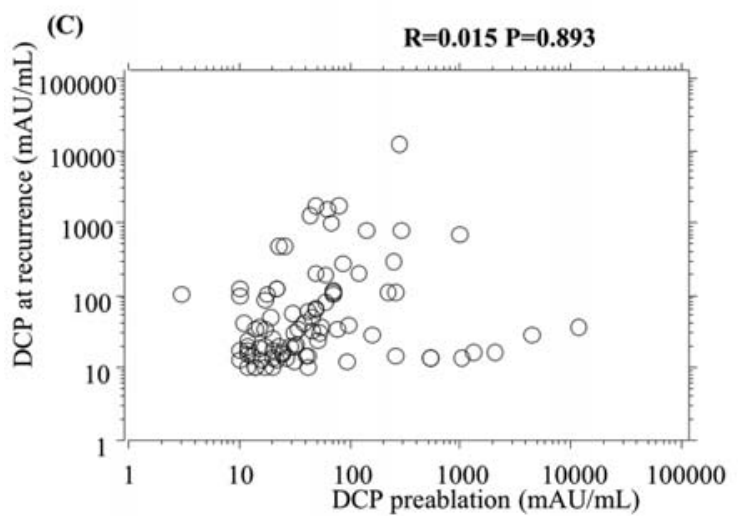

Figure 3. Scatter plots of (A) $\alpha$-fetoprotein, (B) Lens culinaris agglutinin-reactive fraction AFP, (C) des- $\gamma$-carboxy prothrombin pre-ablation and at recurrence. AFP, $\alpha$-fetoprotein; AFP-L3, Lens culinaris agglutinin A-reactive fraction of $\alpha$-fetoprotein; DCP, des- $\gamma$-carboxy prothrombin.

on univariate analysis. Table IV shows the predictive factors for cumulative recurrence-free survival after RFA according to Cox proportional hazards regression; low Alb (Alb $\leq 3.5 \mathrm{~g} /$ dl) levels $(\mathrm{HR}=2.821,95 \% \mathrm{CI} 1.359-5.857 \mathrm{P}=0.0054)$ and high AFP-L3 (AFP-L3 $\geq 10 \%$ ) levels ( $\mathrm{HR}=2.138,95 \% \mathrm{CI}$ 1.087-4.207 $\mathrm{P}=0.0277)$ were the risk factors. High AFP-L3 levels (AFP-L3 $\geq 10 \%$ ) had significantly lower recurrencefree survival rate than low levels on multivariate analysis $(\mathrm{P}=0.0277)$, whereas the other two markers levels did not show association.

\section{Discussion}

The local progression rate following RFA alone has been previously reported to be as high as $17-35 \%$ over a mean follow-up period of 16-25.7 months (10-12). The local recurrence rate after RFA in this study was considerably lower (3.5\% at 5 years) compared to previous reports, even though $23(21 \%)$ of patients had HCCs larger than $30 \mathrm{~mm}$ in diameter. Real-time CT guidance is very useful for ensuring precise positioning of electrode insertion into the HCC nodules even if the lesion is located in the subcapsular or subphrenic region, and it is thus useful for obtaining sufficient ablation margins (30). Despite good control of treated lesions, new tumors frequently occur in the different regions of the liver. Previous studies have reported intra-hepatic distant recurrence rates of $18.7-22.5 \%, 62.1-67 \%$ and $81-81.7 \%$ at 1 , 3 and 5 years, respectively, after percutaneous ethanol injection therapy (PEIT) (31-33), 38 and $60 \%$ at 1 and 2 years, respectively, after RFA (34), and 18 and 52\% at 1 and 2 years, respectively, after RFA or microwave coagulation therapy (35). In the present subjects, distant recurrence occurred in $8.3,19.3$ and $66.3 \%$ at 1,3 and 5 years, respectively, after the combination of TACE and RFA; these rates were comparable to those reported previously (31-35). The recurrences of HCC decrease liver function and worsen prognosis. Therefore, it is important to detect and treat HCC recurrence in the early stage to improve prognosis.

Three tumor markers (AFP, AFP-L3, DCP) have been used in the diagnosis of HCCs (14-20). The measurement of the levels of these three tumor markers provides additional important information for the management of patients with HCC. However, positivity rates of these three tumor markers for small HCCs less than $20 \mathrm{~mm}$ in diameter are as low as $20-40 \%$ and are thus unsatisfactory (27). Over $30 \%$ of patients with small $\mathrm{HCC}$ are positive for none of these three markers (27).

On the other hand, there are few reports concerning the significance and clinical utility of these tumor markers for determining the risk of and detecting recurrent HCCs.

At first, we determined the cut-off levels for the three tumor markers. AFP is currently the most widely used marker for diagnosing and monitoring the development of HCC $(15,22,36)$. It has a sensitivity of $39-65 \%$, a specificity of $76-$ $94 \%$ and a positive predictive value of $9-50 \%$ (29). The higher the AFP cut-off level, the higher the specificity and the lower the sensitivity (37-39). We set the cut-off level of AFP as $100 \mathrm{ng} / \mathrm{ml}$, based on a review of several papers 
(A)

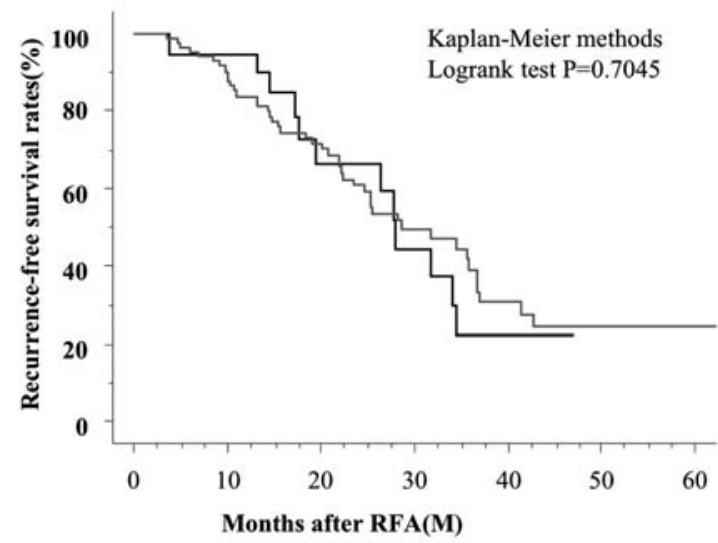

\begin{tabular}{cccccc} 
& 12 & 24 & 36 & 48 & $(\mathrm{M})$ \\
\hline AFP $\geq 100 \mathrm{ng} / \mathrm{ml}$ & 90.0 & 66.6 & 22.2 & 22.2 & $(\%)$ \\
& 84.4 & 60.8 & 38.9 & 24.4 & $(\%)$
\end{tabular}

(C)

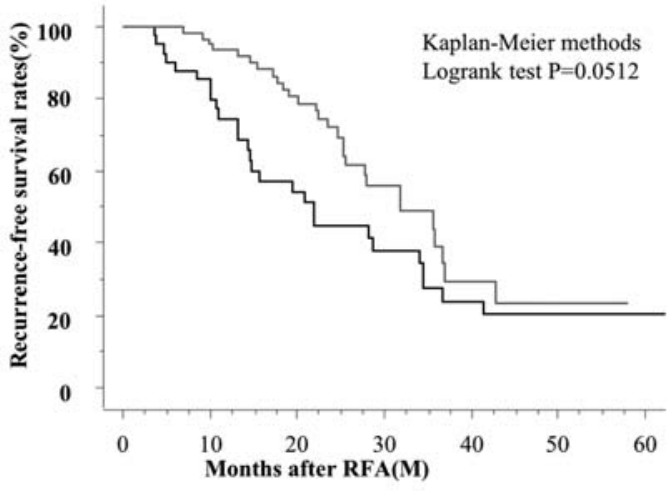

\begin{tabular}{lccccc} 
& 12 & 24 & 36 & 48 & $(\mathrm{M})$ \\
\hline DCP $\geq 40 \mathrm{mAU} / \mathrm{ml}$ & $\mathbf{7 4 . 4}$ & 44.6 & 27.4 & 20.6 & $(\%)$ \\
DCP $<40 \mathrm{mAU} / \mathrm{ml}$ & 93.5 & 72.0 & 38.9 & 23.3 & $(\%)$
\end{tabular}

$(9,15,28,29)$ that reported that this level is suitable for detecting HCCs. In order to improve the specificity of AFP in the diagnosis of HCC, AFP-L3 has been developed. AFP-L3 levels had sensitivities and specificities that ranged from $36-96 \%$ and $89-94 \%$, respectively, though for the diagnosis of small HCCs, the specificity is relatively low, around $20 \%(20,28)$. Pre-treatment AFP-L3 values have also been reported to be correlated with poorly differentiated cancer and poor prognosis $(40,41)$. Another common tumor marker for HCC is PIVKA II, also known as DCP, which results from an acquired defect in the post-translational carboxylation of the prothrombin precursor in HCC cells. Using DCP levels, sensitivities for detecting HCC ranged from 23-57\%. Overall, AFP and DCP have nearly equal sensitivity, although DCP has higher specificity (23-26). We set the cut-off level of AFP-L3 and DCP as $10 \%$ and $40 \mathrm{mAU} / \mathrm{ml}$, respectively, based on previous reports $(20,23-26,28,40,41)$.

In our subjects, the pretreatment AFP, AFP-L3 and DCP values were positive in 19,23 and $41 \%$ of patients, respectively. The positivity rates of AFP and AFP-L3 seemed to
(B)

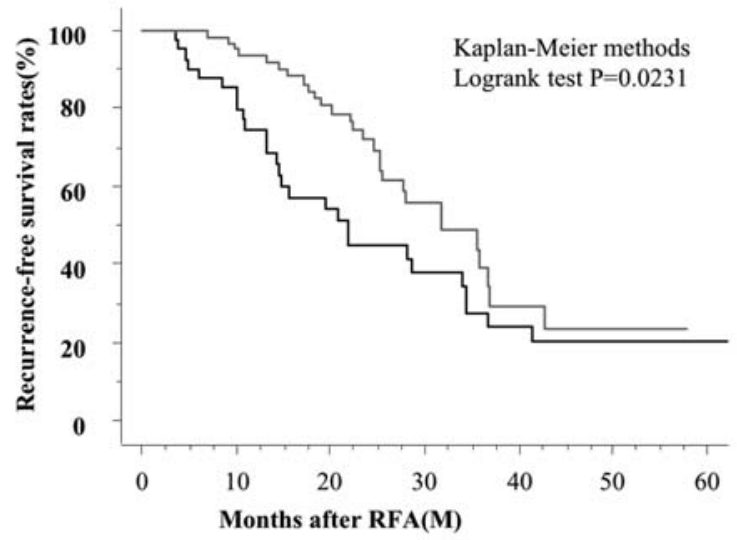

\begin{tabular}{cccccc} 
& 12 & 24 & 36 & 48 & $(\mathrm{M})$ \\
\hline AFP-L3 $210 \%$ & 71.6 & 41.9 & 22.3 & 11.2 & $(\%)$ \\
AFP-L3<10\% & 91.3 & 67.8 & 37.7 & 26.5 & $(\%)$
\end{tabular}

Figure 4. Cumulative recurrence-free survival rates by elevation of pretreatment (A) $\alpha$-fetoprotein (AFP) in patients who underwent the combination of chemoembolization and RFA. There is no significant difference between patients with or without elevated pretreatment AFP levels $(\mathrm{P}=0.7045)$. Cumulative recurrence-free survival rates by elevation of pretreatment (B) Lens culinaris agglutinin A-reactive fraction of AFP (AFP-L3) in patients who underwent the combination of chemoembolization and RFA. The recurrence-free survival rate is significantly lower in patients with than without elevated pretreatment AFP-L3 levels $(\mathrm{P}=0.0231)$. Cumulative recurrence-free survival rates by elevation of pretreatment (C) des- $\gamma$-carboxy prothrombin (DCP) in patients who underwent the combination of chemoembolization and RFA. There is no significant difference between patients with and without elevated pretreatment DCP levels $(\mathrm{P}=0.0512)$.

be low, because $35 \%$ of HCCs are smaller than $20 \mathrm{~mm}$ in diameter. Forty-seven (44\%) patients showed no elevations of any tumor markers, indicating that many RFA-treatable patients showed no tumor marker positivity. These results indicate the limitations of using tumor markers for detecting HCC.

Next, we analyzed these three tumor markers for detecting recurrent HCCs. The rate of AFP, AFP-L3 and DCP positivity did not increase in either the first or the second recurrence of HCCs. Previously it was reported that the AFP-L3-positive rate increased with recurrence. This may suggest that the diagnostic value of AFP-L3 increases during the clinical course of HCC (41). However, the positivity rates for the three makers did not increase significantly with the first or the second recurrence.

The changes of each tumor marker and tumor marker positivity in each patient were examined according to recurrence stages. Surprisingly, no correlation was found for any of the markers between the pre-ablation values and the values at recurrence. Patients who were positive for AFP, AFP-L3 and DCP at the initial HCC did not always have up-regulation of the tumor markers at recurrence. Inversely, patients who 
Table III. Predictive factors for recurrence-free survival (univariate analysis).

\begin{tabular}{|c|c|c|c|}
\hline \multirow[b]{2}{*}{ Variable } & \multicolumn{3}{|c|}{$\begin{array}{l}\text { Cumulative probability of } \\
\text { recurrence-free survival (\%) }\end{array}$} \\
\hline & 2 years & 4 years & P-value \\
\hline \multicolumn{4}{|l|}{ Age } \\
\hline$<67$ & 63.8 & 22.6 & 0.8863 \\
\hline$\geq 67$ & 60.3 & 23.0 & \\
\hline \multicolumn{4}{|l|}{ Gender } \\
\hline Male & 60.5 & 23.2 & 0.9676 \\
\hline Female & 66.1 & 23.4 & \\
\hline \multicolumn{4}{|l|}{ Size of tumor } \\
\hline$<25 \mathrm{~mm}$ & 85.6 & 20.4 & 0.9091 \\
\hline$\geq 25 \mathrm{~mm}$ & 64.9 & 23.9 & \\
\hline \multicolumn{4}{|l|}{ AST } \\
\hline$<52$ IU/1 & 69.2 & 27.3 & 0.113 \\
\hline$\geq 52 \mathrm{IU} / 1$ & 54.9 & 19.3 & \\
\hline \multicolumn{4}{|l|}{ ALT } \\
\hline$<45 \mathrm{IU} / 1$ & 65.5 & 25.1 & 0.4606 \\
\hline$\geq 45 \mathrm{IU} / 1$ & 58.2 & 22.7 & \\
\hline \multicolumn{4}{|l|}{ Alb } \\
\hline$>3.5 \mathrm{~g} / \mathrm{dl}$ & 76.1 & 34.7 & 0.0039 \\
\hline$\geq 3.5 \mathrm{~g} / \mathrm{dl}$ & 49.6 & 13.1 & \\
\hline \multicolumn{4}{|l|}{ T-Bil } \\
\hline$<0.8 \mathrm{mg} / \mathrm{dl}$ & 62.2 & 22 & 0.6158 \\
\hline$\geq 0.8 \mathrm{mg} / \mathrm{dl}$ & 61.6 & 25.4 & \\
\hline \multicolumn{4}{|l|}{ PT } \\
\hline$\geq 84 \%$ & 68.1 & 30.5 & 0.1021 \\
\hline$<84 \%$ & 55.6 & 15.9 & \\
\hline \multicolumn{4}{|l|}{ Plt } \\
\hline$\geq 10 \times 10^{4} / \mathrm{mm}^{3}$ & 64 & 35.2 & 0.2086 \\
\hline$<10 \times 10^{4} / \mathrm{mm}^{3}$ & 59.7 & 14.6 & \\
\hline \multicolumn{4}{|l|}{ AFP pre-ablation } \\
\hline$<100 \mathrm{ng} / \mathrm{ml}$ & 60.8 & 24.4 & 0.7045 \\
\hline$\geq 10 \mathrm{ng} / \mathrm{ml}$ & 66.6 & 22.2 & \\
\hline \multicolumn{4}{|c|}{ AFP-L3 pre-ablation } \\
\hline$<10 \%$ & 67.8 & 26.5 & 0.0231 \\
\hline$\geq 10 \%$ & 41.9 & 11.2 & \\
\hline \multicolumn{4}{|l|}{ DCP pre-ablation } \\
\hline$<40 \mathrm{mAU} / \mathrm{ml}$ & 72 & 23.3 & 0.0512 \\
\hline$\geq 40 \mathrm{mAU} / \mathrm{ml}$ & 44.6 & 20.6 & \\
\hline
\end{tabular}

Alb, albumin; AFP-L3, Lens culinaris is agglutinin A-reactive fraction of $\alpha$-fetoprotein; AFP, $\alpha$-fetoprotein; DCP, des- $\gamma$-carboxy prothrombin; T-Bil, total bilirubin; AST, aspirate aminotransferase; ALT, alanine aminotransferase; PT, prothrombin time; Plt, platelet counts.
Table IV. Predictive factors for recurrence-free survival (multivariate analysis).

\begin{tabular}{lccc}
\hline & $\begin{array}{c}\text { Relative } \\
\text { risk }\end{array}$ & $95 \% \mathrm{CI}$ & P-value \\
\hline $\mathrm{Alb} \leq 3.5 \mathrm{~g} / \mathrm{dl}$ & 2.821 & $1.359-5.857$ & 0.0054 \\
$\mathrm{AFP}-\mathrm{L} 3 \geq 10 \%$ & 2.138 & $1.087-4.207$ & 0.0277 \\
$\mathrm{DCP} \geq 40 \mathrm{mAU} / \mathrm{ml}$ & 1.751 & $0.965-3.174$ & 0.0653 \\
$\mathrm{AST}>52 \mathrm{IU} / \mathrm{l}$ & 1.991 & $0.857-4.628$ & 0.1093 \\
$\mathrm{Plt}<10 \times 10^{4} / \mathrm{mm}^{3}$ & 1.122 & $0.542-2.323$ & 0.7588 \\
$\mathrm{PT}<84 \%$ & 1.117 & $0.529-2.361$ & 0.7711 \\
$\mathrm{ALT}>45 \mathrm{IU} / \mathrm{ml}$ & 0.921 & $0.377-2.242$ & 0.8542 \\
\hline
\end{tabular}

Alb, albumin; AFP-L3, Lens culinaris is agglutinin A-reactive fraction of $\alpha$-fetoprotein; DCP, des- $\gamma$-carboxy prothrombin; AST, aspirate aminotransferase; ALT, alanine aminotransferase; PT, prothrombin time; Plt, platelet counts.

were negative for AFP, AFP-L3 and DCP at the initial HCC did not always have tumor marker values in the normal range at recurrence. Presumably, these facts are due to changes in the properties and degree of differentiation of HCC cells, because HCC cells are very heterogeneous; multicentric occurrence is another possibility that may cause these discrepancies. Based on these results, these three tumor markers appear to be independently increased and all need to be measured to screen for HCC recurrence after RFA. The measurement of the levels of the three tumor markers provides additional important information.

Since local control is excellent, multicentric recurrence was more dominant in more than half of the cases of intrahepatic recurrence in our subjects (42). Kumada et al (43) determined that $50.9 \%$ of recurrent tumors were multicentric in origin, and that $49.1 \%$ were intrahepatic metastases. Sugimoto et al (44) estimated that about $45 \%$ of recurrent tumors were of multicentric origin, with the others being intrahepatic metastases, based on either imaging findings or the above morphologic criteria. The present results suggest that multicentric recurrence is more prevalent after RFA.

Next, we examined the significance of tumor markers in recurrence-free survival and prediction of HCCs. Our study demonstrated that the cumulative recurrence-free rates after 2 and 4 years were independent of AFP and DCP positivity. However, the cumulative recurrence-free rates were significantly worse in patients with AFP-L3 $\geq 10 \%$ than in patients with AFP-L3 $<10 \%$. Pre-treatment AFP-L3 values have also been reported to be correlated with poorly differentiated cancer and poor prognosis $(40,41)$. HCC patients positive for AFP-L3 (AFP-L3 $\geq 10 \%$ ) have the potential for faster growth and early metastasis (45). The elevation of pretreatment AFP-L3 levels significantly decreased the survival rate of patients who underwent RFA (21). Our results suggest that elevated AFP-L3 levels reflect faster distant recurrence of HCCs after RFA.

Then, we examined several significant risk factors for disease-free survival and found that low Alb $(\leq 3.5 \mathrm{mg} / \mathrm{dl})$ 
and high AFP-L3 ( $\geq 10 \%$ ) were predictive factors for cumulative recurrence-free survival after RFA on univariate analysis. The risk factors for tumor recurrence-free survival after curative resection were reported to include not only tumorrelated factors, such as tumor size, nodule number and tumor location, but also liver-related factors, such as platelet count, prothrombin time, serum ALT and serum Alb $(1,6,46,48)$. The present study demonstrated that elevated tumor marker levels, especially elevated AFP-L3 levels, were also risk factors for recurrence after complete RFA. Multivariate analysis demonstrated that low Alb levels and high AFP-L3 levels were independent predictive factors for the cumulative recurrence-free survival rate.

In conclusion, tumor markers have almost the same utility for the detection of recurrent HCCs after RFA as for detection of initial HCCs. Therefore, it is strongly recommended that all three markers be measured to predict and detect HCC recurrence following RFA. Especially high AFP-L3 levels should be followed closely. In addition, other new markers will be needed to establish a more efficient strategy for screening, for early detection and for prediction of HCC recurrence.

\section{References}

1. Yamashiki N, Yoshida H, Tateishi R, et al: Recurrent hepatocellular carcinoma has an increased risk of subsequent recurrence after curative treatment. J Gastroenterol Hepatol Dec 22: 2155-2160, 2007.

2. Parkin DM, Bray F, Ferlay $\mathrm{J}$ and Pisani P: Global cancer statistics, 2002. CA Cancer J Clin 55: 74-108, 2005.

3. Shiratori Y, Yoshida H and Omata M: Management of hepatocellular carcinoma: advances in diagnosis, treatment and prevention. Exp Rev Anticancer Ther 1: 277-290, 2001.

4. Yamakado K, Nakatsuka A, Takaki H, et al: Early-stage hepatocellular carcinoma: radiofrequency ablation combined with chemoembolization versus hepatectomy. Radiology 247: 260-266, 2008.

5. Rossi S, Di Stasi M, Buscarini E, et al: Percutaneous radiofrequency interstitial thermal ablation in the treatment of small hepatocellular carcinoma. Cancer J Sci Am 1: 73-81, 1995.

6. Fuke H, Sugimoto K, Shiraki K, et al: Predictive factors for distant recurrence of $\mathrm{HCV}$-related hepatocellular carcinoma after radiofrequency ablation combined with chemoembolization. Aliment Pharmacol Ther 27: 1253-1260, 2008.

7. Yamakado K, Naktsuka A, Akeboshi M and Takeda K: Percutaneous radiofrequency ablation of liver neoplasms adjacent to the gastrointestinal tract after balloon catheter interposition. J Vasc Interv Radiol 14: 1184-1186, 2003.

8. Yamakado K, Nakatsuka A, Akeboshi M, Takaki H and Takeda K: Percutaneous radiofrequency ablation for the treat-ment of liver neoplasms in the caudate lobe left of the vena cava: electrode placement through the left lobe of the liver under CT-fluoroscopic guidance. Cardiovasc Intervent Radiol 28: 638-640, 2005.

9. Tateishi R, Shiina S, Yoshida H, et al: Prediction of recurrence of hepatocellular carcinoma after curative ablation using three tumor markers. Hepatology 44: 1518-1527, 2006.

10. Vivarelli M, Guglielmi A, Ruzzenente A, et al: Surgical resection versus percutaneous radiofrequency ablation in the treatment of hepatocellular carcinoma on cirrhotic liver. Ann Surg 240: 102-107, 2004.

11. Hong SN, Lee SY, Choi MS, et al: Comparing the outcomes of radiofrequency ablation and surgery in patients with a single small hepatocellular carcinoma and well-preserved hepatic function. J Clin Gastroenterol 39: 247-252, 2005.

12. Montorsi M, Santambrogio R, Bianchi P, et al: Survival and recurrences after hepatic resection or radiofrequency for hepatocellular carcinoma in cirrhotic patients: a multivariate analysis. J Gastrointest Surg 9: 62-67, 2005.

13. Zhou L, Liu J and Luo F: Serum tumor markers for detection of hepatocellular carcinoma. World J Gastroenterol 12: 1175-1181, 2006.
14. Toyoda H, Kumada T, Osaki Y, Oka H and Kudo M: Role of tumor markers in assessment of tumor progression and prediction of outcomes in patients with hepatocellular carcinoma. Hepatol Res 37 (Suppl. 2): S166-S171, 2007.

15. Oka H, Tamori A, Kuroki T, Kobayashi K and Yamamoto S: Prospective study of alpha-fetoprotein in cirrhotic patients monitored for development of hepatocellular carcinoma. Hepatology 19: 61-66, 1994.

16. Taketa K, Endo Y, Sekiya C, et al: A collaborative study for the evaluation of lectin-reactive alpha-fetoprotein in early detection of hepatocellular carcinoma. Cancer Res 53: 5419-5423, 1993.

17. Mita Y, Aoyagi Y, Yanagi M, Suda T, Suzuki Y and Asakura H: The usefulness of determining des-gamma-carboxy prothrombin by sensitive enzyme immunoassay in the early diagnosis of patients with hepatocellular carcinoma. Cancer 82: 1643-1648, 1998.

18. Hippo Y, Watanabe K, Watanabe A, et al: Identification of soluble NH2-terminal fragment of glypican-3 as a serological marker for early-stage hepatocellular carcinoma. Cancer Res 64: 2418-2423, 2004.

19. Tsai JF, Jeng JE, Chuang LY, et al: Serum insulin-like growth factor-II as a serologic marker of small hepatocellular carcinoma. Scand J Gastroenterol 40: 68-75, 2005.

20. Marrero JA and Lok AS: Newer markers for hepatocellular carcinoma. Gastroenterology 127: S113-S119, 2004.

21. Toyoda H, Kumada T, Kaneoka Y, et al: Prognostic value of pretreatment levels of tumor markers for hepatocellular carcinoma on survival after curative treatment of patients with HCC. J Hepatol 49: 223-232, 2008.

22 Tsukuma H, Hiyama T, Tanaka S, et al: Risk factors for hepatocellular carcinoma among patients with chronic liver disease. N Engl J Med 328: 1797-1801, 1993.

23. Taketa K, Sekiya C, Namiki N, et al: Lectin-reactive profiles of alpha-fetoprotein characterizing hepatocellular carcinoma and related conditions. Gastroenterology 99: 508-518, 1990.

24. Liebman HA, Furie BC, Tong MJ, et al: Des-gamma-carboxy (abnormal) prothrombin as a serum marker of primary hepatocellular carcinoma. N Engl J Med 310: 1427-1431, 1984.

25. Fujiyama S, Tanaka M, Maeda S, Ashihara H, Hirata R and Tomita K: Tumor markers in early diagnosis, follow-up and management of patients with hepatocellular carcinoma. Oncology 62: S57-S63, 2002.

26. Toyoda H, Kumada T, Kiriyama S, et al: Prognostic significance of simultaneous measurement of three tumor markers in patients with hepatocellular carcinoma. Clin Gastroenterol Hepatol 4: 111-117, 2006.

27. Shiraki K and Takase K: New serological markers for hepatocellular carcinoma. Hepatol Res 33: 195-197, 2005.

28. Snowberger N, Chinnakotla S, Lepe RM, et al: Alpha fetoprotein, ultrasound, computerized tomography and magnetic resonance imaging for detection of hepatocellular carcinoma in patients with advanced cirrhosis. Aliment Pharmacol Ther 26: 1187-1194, 2007.

29. Daniele B, Bencivenga A, Megna AS and Tinessa V: Alphafetoprotein and ultrasonography screening for hepatocellular carcinoma. Gastroenterology 127 (Suppl. 1): S108-S112, 2004.

30. Yamakado K, Nakatsuka A, Ohmori S, et al: Radiofrequency ablation combined with chemoembolization in hepatocellular carcinoma: treatment response based on tumor size and morphology. J Vasc Interv Radiol 13: 1225-1232, 2002.

31. Hasegawa S, Yamasaki N, Hiwaki T, et al: Factors that predict intrahepatic recurrence of hepatocellular carcinoma in 81 patients initially treated by percutaneous ethanol injection. Cancer 86: 1683-1690, 1999.

32. Ebara M, Okabe S, Kita K, et al: Percutaneous ethanol injection for small hepatocellular carcinoma: therapeutic efficacy based on 20-year observation. J Hepatol 43: 458-464, 2005.

33. Khan KN, Yatsuhashi H, Yamasaki K, et al: Prospective analysis of risk factors for early intrahepatic recurrence of hepatocellular carcinoma following ethanol injection. J Hepatol 32: 268-278, 2000.

34. Horiike N, Iuchi $\mathrm{H}$, Ninomiya $\mathrm{T}$, et al: Influencing factors for recurrence of hepatocellular carcinoma treated with radiofrequency ablation. Oncol Rep 9: 1059-1062, 2002.

35. Izumi N, Asahina Y, Noguchi O, et al: Risk factors for distant recurrence of hepatocellular carcinoma in the liver after complete coagulation by microwave or radiofrequency ablation. Cancer 91: 949-956, 2001. 
36. Ikeda K, Saitoh S, Koida I, et al: A multivariate analysis of risk factors for hepatocellular carcinogenesis: a prospective observation of 795 patients with viral and alcoholic cirrhosis. Hepatology 18: 47-53, 1993.

37. Sherman M, Peltekian KM and Lee C: Screening of hepatocellular carcinoma in chronic carriers of hepatitis B virus: incidence and prevalence of hepatocellular carcinoma in a North American urban population. Hepatology 22: 432-438, 1995.

38. Trevisani F, D'Intino PE, Morselli-Labate AM, et al: Serum $\alpha-$ fetoprotein for diagnosis of hepatocellular carcinoma in patients with chronic liver disease: influence of HbsAg and anti-HCV status. J Hepatol 34: 570-575, 2001.

39. Gambarin-Gelwan M, Wolf DC, Shapiro R, Schwartz ME and Min AD: Sensitivity of commonly available screening tests in detecting hepatocellular carcinoma in cirrhotic patients undergoing liver transplantation. Am J Gastroenterol 95: 1535-1538, 2000 .

40. Oka H, Saito A, Ito K, et al: Multicenter prospective analysis of newly diagnosed hepatocellular carcinoma with respect to the percentage of Lens culinaris agglutinin-reactive alphafetoprotein. J Gastroenterol Hepatol 16: 1378-1383, 2001.

41. Yamashita F, Tanaka M, Satomura S and Tanikawa K: Prognostic significance of Lens culinaris agglutinin A-reactive alpha-fetoprotein in small hepatocellular carcinomas. Gastroenterology 111: 996-1001, 1996.
42. Nakashima O and Kojiro M: Recurrence of hepatocellular carcinoma: multicentric occurrence or intrahepatic metastasis? A viewpoint in terms of pathology. J Hepatobiliary Pancreat Surg 8: 404-409, 2001.

43. Kumada T, Nakano S, Takeda I, et al: Patterns of recurrence after initial treatment in patients with small hepatocellular carcinoma. Hepatology 25: 87-92, 1997.

44. Sugimoto R, Okuda K, Tanaka M, Aoyagi S and Kojiro M: Metachronous multicentric occurrence of hepatocellular carcinoma after surgical treatment - clinicopathological comparison with recurrence due to metastasis. Oncol Rep 6: 1303-1308, 1999.

45. Debruyne EN and Delanghe JR: Diagnosing and monitoring hepatocellular carcinoma with alpha-fetoprotein: new aspects and applications. Clin Chim Acta 395: 19-26, 2008.

46. Ng KK, Poon RT, Lo CM, Yuen J, Tso WK and Fan ST: Analysis of recurrence pattern and its influence on survival outcome after radiofrequency ablation of hepatocellular carcinoma. J Gastrointest Surg 12: 183-191, 2008.

47. Kobayashi M, Ikeda K, Kawamura Y, et al: High serum desgamma-carboxy prothrombin level predicts poor prognosis after radiofrequency ablation of hepatocellular carcinoma. Cancer 115: 571-580, 2009. 